\title{
Starburst dwarfs - fueling and morphological evolution
}

\author{
Nils Bergvall ${ }^{1}$, Göran Östlin ${ }^{2}$, Josefa Masegosa ${ }^{3}$, Erik Zackrisson ${ }^{1}$ \\ 1) Astronomical observatory, Box 515, S-75120 Uppsala, Sweden; 2) Institut \\ d'Astrophysique de Paris, 98bis Boulevarrd Arago, F-75014 Paris, France; 3) \\ Instituto de Astrofisica de Andalucia, CSIC, Apdo. 3004, 18080, Granada, Spain
}

Sept 10, 1999

\begin{abstract}
The effects of mergers in low mass galaxies are poorly understood. In this paper we analyze different observational evidences which support the view that mergers can trigger starbursts in dwarf galaxies. We discuss the relationships between blue compact galaxies (BCGs), dIs, dEs and low surface brightness galaxies (LSBGs) and present some evidences which favour that strong starbursts are caused by mergers between dEs and LSBGs.
\end{abstract}

\section{Introduction}

Since Zwickys (1964) early discovery of Blue Compact Galaxies (BCGs) a large amount of work has been devoted to investigate their nature. Major questions are what causes the burst, how long it can be sustained and if the heating and mechanical energy it produces eventually will cause the cas to be expelled from the system. Recent models (Mac Low and Ferrara (1999)) indicate that the galaxy probably can retain its gas if $\mathcal{M} \geq 10^{7} \mathcal{M}_{\odot}$. Cooling and reaccretion may later ignite a new burst and in a Hubble time a few such bursts may occur. Another possibility is the young galaxy hypothesis. Today we have two hot candidates, IZw18 and SBS0335-052, both with extremely low metallicities. The possible presence of old stars is still debated however (Aloisi et al. (1999); Hunter \& Thronson (1996); Papaderos et al. (1996)). In both cases signs of merger activity are evident. This also seems to be a common property of the most active starburst dwarfs and may be the most likely scenario to explain the burst activity.

\section{Morphology and colours. The starburst progenitors}

Different classification systems have been proposed for starburst dwarfs. Telles et al. (1997) separate two different types, one regular type and the other more luminous, having an irregular structure. These may be related, modified in their morphology by evolution.

In the merger scenario one of the progenitors has to be a gas rich, metal poor galaxy, i.e. a dI galaxy, a Low Surface Brightness Galaxy 
(LSBG) or an isolated HI cloud. We have studied the global properties of a few LSBGs and BCGs to look for possible relationships and find that the global parameters like disk scale length and central surface brightness are quite similar. It has been claimed (Papaderos et al. (1996)) that LSBGs cannot be related to BCGs since these two parameters differ significantly. However, the problem may be related to the lack of very deep images. The scalelengths are derived from regions too close to the central starburst. Fig. 1 from a deep B image of ESO 338-IG04 shows that the slope of the profile changes at $\mu_{B} \sim 27^{m}$ $\operatorname{arcsec}^{-2}$. An exponential fit to the outer profile results in a central surface brightness of $\mu_{B, 0} \approx 24.5$, typical of a faint LSBG. The difference between a power law fit and an exponential fit is however minor and we need to look at the colours of the halo to determine what type of galaxy may be involved. Fig. 2 shows results from surface photometry in the optical/near-IR of the BCG halos and the global colours of our sample of blue LSBGs compared to predictions from our spectral evolutionary models. For comparison the colours of the halo of IZw18 (Östlin et al., in prep.) is also indicated. All LSBGs are starforming so the short burst model does not apply. Thus the ages are $\geq 2-3$ Gyr. Although we cannot go as far out into the halo in the near-IR as in the optical, we find that the halo colours of the BCGs range from those of LSBGs to those of very old red stellar populations. One of the galaxies, ESO 338-IG04, has an exponential LSB disk and at the same time contains a system of globular clusters (Östlin et al. (1998)) with a full range of ages and indications of a few bursts in the past. The GCs indicate the presence of a relatively massive, very old system. A probable scenario is therefore a merger between an LSBG and a dE/dSph. Other strong supports of merger activities come from a study of the $\mathrm{H} \alpha$ velocity field in this galaxy and a handful of other luminous BCGs (Östlin et al. (1999)).

\section{Metallicities and $\mathrm{HI}$ content}

If mergers are the cause of luminous starbursts, why would they be fed by LSBGs and not dIs? Bergvall et al. (1998) argued that LSBGs are more attractive due to the constraints set by the metallicities and the $\mathrm{HI}$ contents of dwarf galaxies in general. Under the assumption that the samples available in the literature are representative, the luminositymetallicity diagram indicates a luminosity difference between a BCG and a dI at the same metallicity of $\sim 2$ magnitudes. A bursting $\mathrm{dI}$

would obtain a $\mathcal{M}_{\mathcal{H I}} / \mathrm{L}_{B}$ drastically lower than what is observed for BCGs. LSBGs have no such problem. Fig. 3 illustrates the situation in 


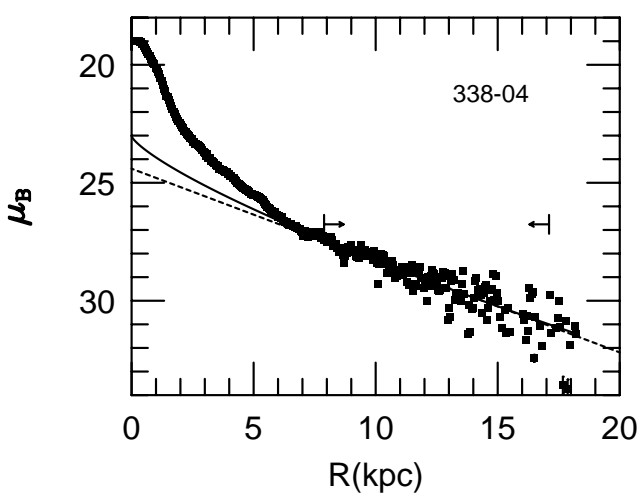

Figure 1. The luminosity profile of ESO 338-IG04 in B, corrected for an inclination of $i=62^{\circ}$. The hatched line is a pure exponential fit and the full drawn line is the best fit to a Sersic law with an exponent of 0.8. (ESO NTT.)

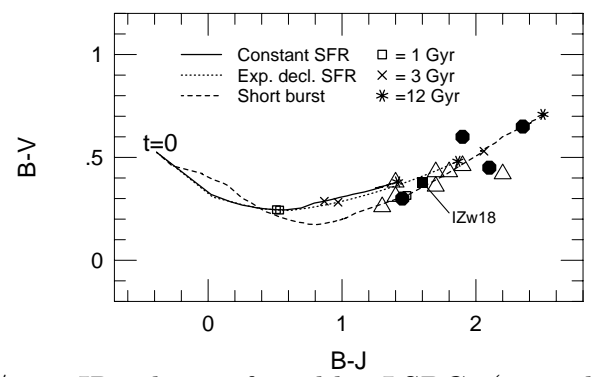

Figure 2. Optical/near-IR colours of our blue LSBGs (triangles) and the halos of the 4 luminous BCGs ESO 338-IG04, ESO 350-IG38, ESO 400-G34 and ESO 480-IG12 (filled dots) and IZw18 (filled square). The predicted colour evolution of three different star forming scenarios is displayed: constant star formation rate (SFR), an exponentially declining SFR and a short initial burst. Data obtained mainly at ESO.

a slightly different way. There seems to be a clear distinction between $\mathrm{dI} /$ Is and LSBGs in the $\mathrm{O} / \mathrm{H}-\mathcal{M}_{\mathcal{H I}}$ diagram. It shows very nicely that they are the same family, the LSBGs being the less evolved and more gas rich ones. If we ignite a starburst, the position of a galaxy would shift towards a lower gas mass and a higher metallicity. This indicates that the best guess progenitors for the burst component of the BCGs are the LSBGs. In the most luminous cases, e.g. ESO 338-04 and ESO 400-43, as for SBS0335, LSBGs seems to be the only option.

The two most metal poor starburst dwarfs, IZw 18 and SBS0335-052, are located in the same region as the LSBGs. Are these two galaxies in an early phase of the merging process? The disturbed HI morphology of IZw 18 (van Zee et al. (1964)) may point to this. The morphology of the HII/HI cloud of SBS0335-052 (see also Pustilnik et al 1999, in 


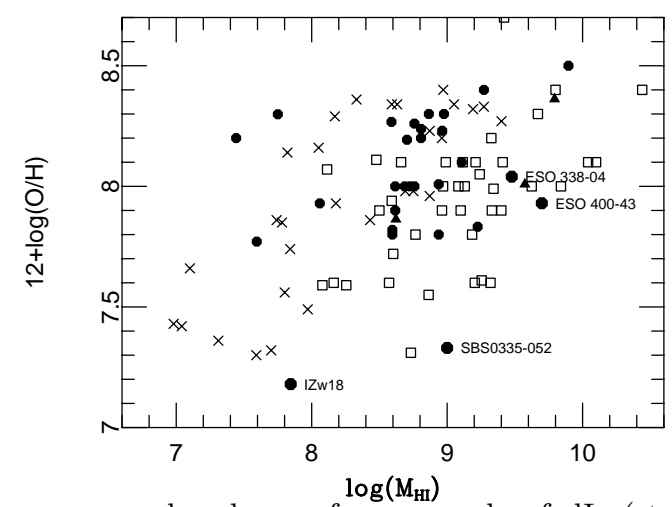

Figure 3. HI mass versus oxygen abundances for a sample of dIs (stars), BCGs (filled dots) and LSBGs (squares) obtained from the literature, including our own data from ESO, Nancay and Parkes.

preparation) also opens the long standing question of the low metallicity. Are we measuring the metallicity of the nearly pristine HI gas cloud illuminated by the starburst or has it something to do with the underlying galaxy?

A possible scenario that would explain the different properties of dIs and BCGs is that the star formation efficiency is ruled by the dark matter potential field. The DM is more centrally concentrated in dEs than in dIs and this may make the difference. Since the DM dominates the mass distribution in dwarf galaxies, the burst we see in luminous BCGs today therefore mimics the first burst in the history of the dE. A merger between an LSBG and a dI would probably be less dramatic.

\section{References}

Aloisi, A., Tosi, M., Greggio, L., 1999, AJ 118, 302

Bergvall N., Östlin G., Pharasyn A., Rönnback J., Masegosa J.: 1998, in Highlights of Astronomy, vol. 11

Hunter, D.A., Thronson, H.A. Jr., 1996, ApJ 461, 202

Mac Low, M.-M., Ferrara, A., 1999, ApJ 513, 42

Östlin, G, Bergvall, N., Rönnback., J., 1998, A\&A 335, 85

Östlin, G, Amram, P., Masegosa, J., Bergvall, N., Boulesteix., J., 1999, A\&AS 137, 419

Papaderos, P., Loose, H.-H., Fricke, K.J., Thuan, T.X., 1998, A\&A 338, 43

Papaderos, P., Loose, H.-H., Thuan, T.X., Fricke, K.J., 1996, A\&AS 120, 207

Telles, E., Melnick, J., Terlevich, R., 1997, MNRAS 288, 78

van Zee, L., Westpfahl, D., Haynes, M.P., Salzer, J., 1998, AJ, 115, 1000

Zwicky, F., 1964, ApJ 140, 1467 